\title{
The Study on Aging Psychology According to the Elderly Care under the Glocalization Perspective
}

\author{
T. Wang and Z. Yu
}

\begin{abstract}
Applying the method of social network analysis, the current study analyzes all the publications from Web of Science since 1990 regarding studies on aging. Previous studies based on the data of Chinese Social Science Citation Index (CSSCI) since 1998(Wang \& Hou, 2016), show that there are some common constructs such as aging, mental health, life satisfaction and subjective well-being in both English and Chinese, while studies considering the life motivation and the role of culture has received little attention in Chinese publications. By contrast, in English publications on Psychology, studies regarding aging have been defined by certain aspects, such as objective surveys rather than inter-subjective investigations, continuous aging rather than merely the older stage, and rigorous case studies rather than action research. However, based on a two-year-practice in a Chinese elderly caring house, the author challenged these presuppositions and proposed an alternative perspective with the approach of glocalization.
\end{abstract}

Index Terms-Topical structure analysis, aging study, glocalization.

\section{INTRODUCTION}

As the number of old adults in China is increasing, the problem of the elderly becomes a high-light topic in Chinese society. One of the previous studies has analyzed the topical Structure of Chinese gerontology based on the data from the Chinese Social Science Citation Index (CSSCI) [1] and found that the influence of culture received little attention on the studies of the elderly in Chinese publications; which might be a very important topic for the aged people living in caring house. Previous studies in English literature consider the impact of culture on the older population, especially on the social motivation and emotions [2]-[4]. Furthermore, the future time perspective, which recently becomes an important variable in aging Psychology [5], has not attracted adequate attention in Chinese literature.

\section{DATA SOURCES AND ANALYTIC PROCEDURE}

The current study has employed the Web of Science sources as method of data collection with regards to all research that has been published in English literature from 1900 until 2018. The key words that were used for each one of the themes were: "aging", "old", and "psychology".

From 1900 until 2018, 1,946 articles written in English were obtained regarding studies of aging in psychology [6].

Manuscript received September 30, 2018; revised November 27, 2018

T. Wang is with the Wuhan University, Wuhan, PRC (e-mail: wangtangsheng@whu.edu.cn).

Z. Yu, born is with the Harrisburg Christian School, PA, USA.
The retrieved data was analyzed applying the method of social network. Firstly, publication institutions, issued years, publication journals and journal distributions of research results were quantitatively analyzed. Secondly, co-words analysis was applied to obtain high frequency keywords and their co-concurrence matrix. The authors imported the co-concurrence matrix into Ucinet, which generated key words clustering a network diagram. Thirdly, the results of social network analysis were discussed according to investigations in a Chinese elderly caring house in order to analyze the aging research theme trend in the future in China, under the Glocalization Perspective.

\section{A. Analysis of Issued Years' Distribution}

Issued years distribution is partly an indicator of the research state, research level, development speed and development stage of a specific research field. This article mainly counts the issued years distribution tendency of domestic research of the old adults' in aging psychology from 1922 to 2018 as shown in Fig. 1. The decision on date limit was made due to lack of evidence in the publications before 1922 in the Web of Science. It is obvious that since 2007 the literature has already transformed into a high-speed development of gerontology and aging psychology research, and reached a climax in 2016.

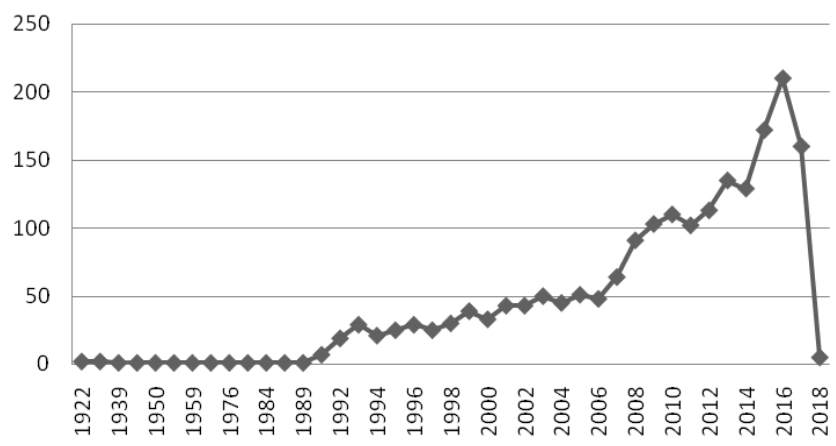

Fig. 1. 1900-2018 aging psychology research annual post trend

As the trends of the Fig. 1 illustrate, periodically English literature was slightly increased and decreased before 2006 . Nevertheless, it has steadily increased year by year from 2007, meaning that the global aging research is increasing from 2007 to the present. It can be inferred that the Chinese studies may be influenced by the degree of population aging, the economic situation, as well as policy issues and the influence of traditional culture in many aspects. By contrast, it could be assumed that China's aging research may be in the embryonic stage of the west before 2005, rather than the maturing period of slow growth [1]. 


\section{B. Institutional Contributions}

Publications' of institution distributions in the Web of Science can generally depict the research strength distribution of domestic studies concerning the old and aging psychology, providing relevant research in institutional cooperation and communication with resources. Among the 1946 articles in the Web of science, there are 100 institutions posting more than 7 articles, and 8 institutions which have published more than 20 articles.

There is no suspense, the United States research institutions occupied the vast majority of the whole world, Max Planck Institute for Human Development, Japan's Tokyo university, one European and one Asian institution ranked fifth and sixth; which rises concerns for Chinese researchers. The production of papers might be influenced by either research resources, or the market needs - while the former keeps being relatively consistent, the latter is changing.

If we combine the publications per institutions and publication years, we can have a better perspective of the Fig. 2 and the developmental tendency.

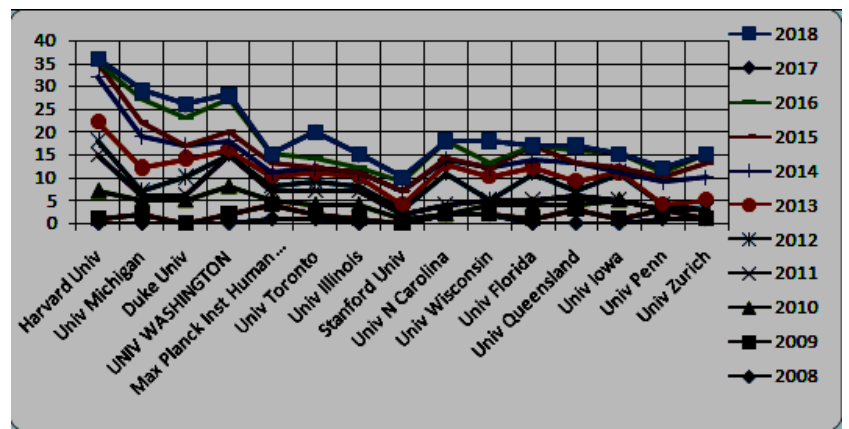

Fig. 2. Annual distribution of years of issued institution on aging psychology.

As the Fig. 2 depicts, for many years - although the overall ranking of Max Planck Inst Human Dev and Stanford University are very high (ranking $5^{\text {st }}$ and $8^{\text {th }}$ ), but during last decade their setbacks are much significant, in contrast, University of Washington, Toronto, Queensland and Zurich perform very powerfully. They are all very good competitors. Supposed that the research resource keeps relatively consistently, it might mainly owe to the market needing. And former research may become a burden to Stanford University and Max Planck Inst Human Dev as well as to Renmin University of China in Chinese literature [1].

\section{Distribution Analysis by Publications per Journal}

There are 12 journals that issued over 15 papers on aging psychology; the ranking list and specific statistical data are shown in Fig. 3. As they clearly demonstrated, Psychology and Aging was the first one. Most of the journals tend to focus on aging psychology in terms of personality and mental health, which are common psychological variables for all the age stages. Surprisingly, the Journal of Experimental Child Psychology is a professional journal which differs greatly from other journals in the object of study. The European Journal of Personality and the Australian Psychologist come out in front out of expection, which implicate different developing speeds in different districts over the global besides USA.

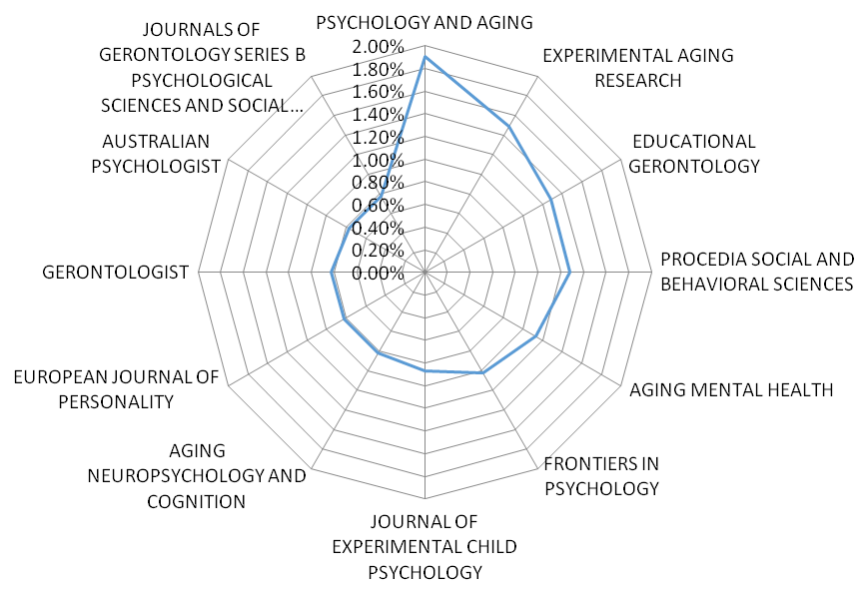

Fig. 3. Publication Journal distribution analysis.

\section{Most Frequently Cited Documents}

As far as the research significance is concerned, additional attention is required by the most frequently cited research papers. Table I shows the top 10 most frequently cited documents in English literature.

TABLE I: TOP 10 MOST CITED RESEARCH PAPERS

(1)Taking time seriously - A theory of socioemotional selectivity. The American psychologist, 1999, Cited 1599 time [7].

(2)Hemispheric asymmetry reduction in older adults: The HAROLD model. Psychology and aging, 2002. Cited 1066 times [8].

(3)Developmental neuropsychology, 1991. Cited 731 times (Welsh, Pennington, \& Groisser, 1991).

(4)Social and emotional patterns in adulthood: Support for socioemotional selectivity theory. Psychology and aging, 1992.Cited 669 times [9].

(5)Time, Human Agency, and Social Change: Perspectives on the Life Course. Social Psychology Quarterly, 1994. Cited 491 times [10].

(6)Diagnosis and treatment of Alzheimer disease and related disorders - Consensus statement of the American Association for Geriatric Psychiatry, the Alzheimer's Association, and the American Geriatrics Society. JAMA-Journal of the American medical, 1997. Cited 656 times [11].

(7)Clinical practice guideline: Treatment of the school-aged child with attention-deficit/hyperactivity disorder. Pediatrics, 2001. Cited 607 times [12].

(8)Enhancing well-being and alleviating depressive symptoms with positive psychology interventions: A practice-friendly meta-analysis. Journal of clinical Psychology, 2009. Cited 554 times [13].

(9)Sensory functioning and intelligence in old-age - A strong connection. Psychology and aging, 1994. Cited 542 times [14].

(10)Lifespan psychology: Theory and application to intellectual functioning. Annual review of Psychology, 1999. Cited 432 times [15]

First of all, the difference in the frequency of citation between the Chinese and English literature is of a significant extent. Secondly, the most highly cited documents in
English literature have been cited for a much longer period of time. Surprisingly, the majority of published research in English, is concerned with child maturing process from 
childhood to adulthood. In this case, the concept of aging means continuity rather than a particular stage, such as old adults. On these grounds, the reason behind this difference might not be the misinterpretation of the studies due to failure in accurate translation. It could potentially be that our culture varies to a degree where it leads, or differentiates, the researchers's concerns.

The culture might play a significant role for this difference. Potentially the western researchers are mostly concerned about the younger generation, and encourage people to "avoid", rather than to face the elderly life. As Laura Carstensen mentioned - one of the authors with great experience of working in hospitals in the United States "Young people have more concerns than the elderly. Hence, the research is mostly focused on the younger rather than the elder". Even though Carstensen focused on the elders, her study still focused on the successful aging for the younger elders rather than the elderly [16].

\section{ANALYSIS OF THE STRUCTURE OF THE THEMES}

Keywords can clarify the themes of each field study and their hierarchical relationship. There are 4610 keywords in 1946 papers. As far as each keyword is counted, the maximum frequency is 184 , and 3694 keywords have 1 frequency.
Goffman argues that in documents there is a critical value which distinguishes high-frequency keywords from low ones, and the high-frequency keywords are the most suited to describe the themes. He thereby put forward the critical point $n=(-1+\sqrt{ } 1+8 \mathrm{I} 1) / 2$, where $n$ is the critical point of word frequency, I1 is the number of 1 frequency word, and $n$ is the critical section midpoint [17]. Defining the upper bound of the critical region with the highest frequency word, and measuring the distance from $\mathrm{n}$ to upper bound on the purpose to define the lower bound of critical region. Then, we got that $\mathrm{n}$ is about 85 , the lower bound of critical region is 1. Because the co-occurrence software limits max numbers of rows and columns to 100 , we can assume that the keyword frequency of lower bound is 9 .

To reveal the core research contents in this field, the authors generated not-less than 9 keywords frequency co-occurrence matrix (101) $(101 * 101)$ by Excel 2010,imported the Ucinet network density and generated the co-occurrence analysis diagram, as shown in Fig. 4.

In this diagram, the connected lines represent the numbers of co-occurrence, while the nods represent the keywords. The more connected the lines are, the higher the numbers of occurrence. Hence, the times of occurrence are shown by line density.

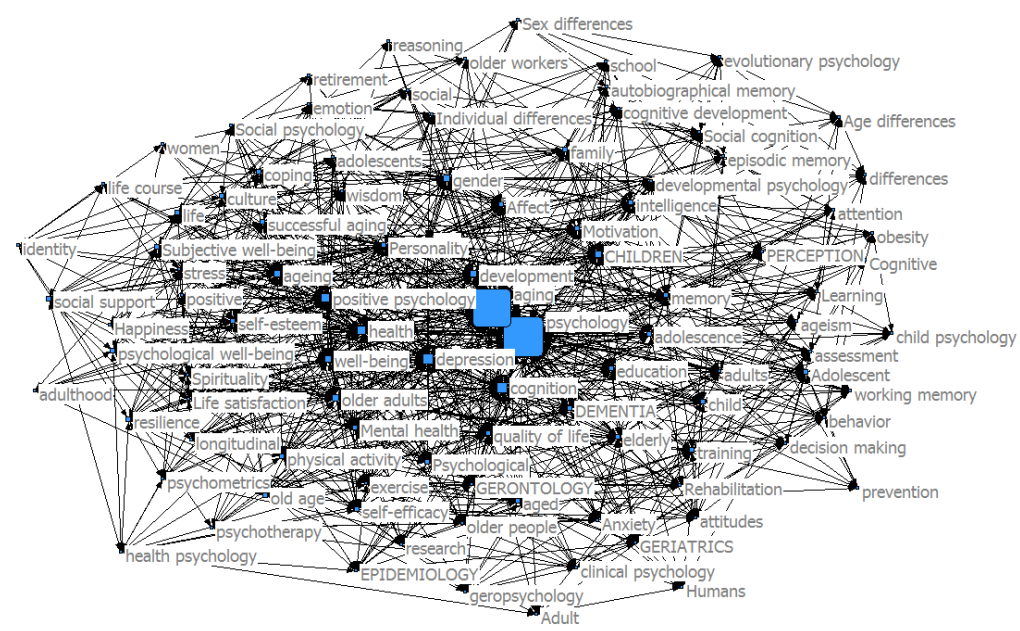

Fig. 4. Keywords density analysis diagram in aging psychological research documents.

Given that the connected lines represent the numbers of co-occurrence, and that the nods represent the keywords, the more the connected lines, the higher the numbers of occurrence. Hence, the times of occurrence are shown by line density. As we can see, in the English literature (Web of Science) study on aging psychology, life satisfaction, subjective well-being, quality of life and mental health are also of primary importance. The study of aging is related to research with children, adolescents and youth, and they are both horizontal and vertical studies. Culture is regarded as a core variable, while the role of the family is also valued. But for the aging psychology, the elderly and elderly unique psychological phenomenon is relatively weak.

TABLE II: The Central Degrees of Aging Psychological Research Documents Keywords

\begin{tabular}{|l|l|l|l|}
\hline & $\begin{array}{l}\text { Freeman's degree } \\
\text { centrality } \\
\text { measures }\end{array}$ & & $\begin{array}{l}\text { Freeman's degree } \\
\text { Centrality } \\
\text { measures }\end{array}$ \\
\hline psychology & 76.768 & life satisfaction & 20.202 \\
\hline aging & 74.747 & adolescence & 20.202 \\
\hline depression & 43.434 & life & 20.202 \\
\hline positive psychology & 42.424 & intelligence & 20.202 \\
\hline health & 42.424 & spirituality & 20.202 \\
\hline
\end{tabular}




\begin{tabular}{|c|c|c|c|}
\hline cognition & 40.404 & old age & 19.192 \\
\hline well-being & 38.384 & gerontology & 18.182 \\
\hline age & 36.364 & social support & 18.182 \\
\hline development & 32.323 & child & 18.182 \\
\hline older adults & 31.313 & stress & 18.182 \\
\hline personality & 27.273 & exercise & 18.182 \\
\hline children & 27.273 & positive & 17.172 \\
\hline gender & 26.263 & adolescents & 17.172 \\
\hline memory & 26.263 & self-efficacy & 17.172 \\
\hline quality of life & 25.253 & training & 17.172 \\
\hline ageing & 24.242 & coping & 17.172 \\
\hline aged & 24.242 & family & 17.172 \\
\hline subjective well-being & 23.232 & longitudinal & 17.172 \\
\hline mental health & 23.232 & wisdom & 17.172 \\
\hline physical activity & 23.232 & Happiness & 16.162 \\
\hline motivation & 22.222 & culture & 16.162 \\
\hline resilience & 22.222 & older people & 15.152 \\
\hline dementia & 22.222 & rehabilitation & 15.152 \\
\hline affect & 22.222 & adults & 15.152 \\
\hline elderly & 22.222 & attitudes & 15.152 \\
\hline self-esteem & 21.212 & adolescent & 15.152 \\
\hline education & 21.212 & social psychology & 14.141 \\
\hline psychological well-being & 21.212 & clinical psychology & 14.141 \\
\hline psychological & 21.212 & cognitive development & 14.141 \\
\hline successful aging & 21.212 & epidemiology & 14.141 \\
\hline
\end{tabular}

The central degrees are listed in Table II. The value is bigger and the key word is closer to the centre. The results clearly show that aging is continuous rather than a particular period. In this study, children, adolescence and the youth are placed in the centre, removing older adults and the elderly at the marginal by comparison.

\section{A FiELD INVESTIGATION IN A CHINESE CARING House}

The authors investigated all the older people in a Chinese geracomium called Rui Fu An in Wuchang. The duration of this research was approximately two year during which in-depth interviews were conducted with 25 older people on a one-to-one basis. From this fieldwork the researcher came to realise that the meaning of life played a very important role for people over age 85 . The oldest people in the caring house used to always ask this kind of questions: 'What do I live for?' What they mean is actually that nobody loves them or can be loved by them. As a consequence, they need a transitional object to show their affection, hope and love. As time goes by, this research collected various data from the same subjects because of inter-subjectivity. The author became more well-informed and the investigation obtained more anti-positive Psychological aging effect; which is not consistent with the viewpoints of socioemotional selectivity theory [9].

Based on the investigation in the caring house, the researcher make the assumption that culture such as in
Guanxi or Qinqing might provide some people with ultimate care. But for the most Chinese investigators, they often put themselves into trouble with the Chinese characteristics for two factors. Firstly, the inter-subjectivity should be considered a confounding variable in modern scientific psychology, rather than a concerning psychological variable. Secondly, Chinese Psychological researchers obey the modern scientific rule to follow a rather global perspective, rather than to develop a Chinese local Psychology,even though relationships with their children might be the most important to Chinese elderly.

What might the elderly's relationships - especially with their children - mean? Firstly, it might links to their Psychosocial age. Secondly, it might raise concerns abouttheir Psychosocial sex. Thirdly, most of them are certainly concerned with the elderly subjective well-being. Finally, and unfortunately, Chinese children are running further and further away from their parents during the glocalization.

Taking the above into consideration, the current study proposes the following topics as potential themes for further research in aging.

(1)What is the meaning of Qinqing or family to the elderly? (2)Is it connected with the spirituality and the meaning of the life?

(3)Should the relationships with their children be a key psychological variable for the elderly in the view of glocalization? 


\section{CONCLUSION AND DiscUSSION}

To sum up, the current study uses bibliometrics technology to directly reveal the development status quo and trends of gerontology and aged research in the last decade: from the issuing institution, the existing research power is concentrated on a few colleges, universities, scientific research institutions; from the point of the issued year, current elderly and aging psychology research have far away entered the mature stage where publications grow slowly; from the point of periodicals, geriatric research mainly concentrated on the field of aging psychology.

According to the main themes in English literature, aging study is defined by some settings in aging models, such as objective surveys rather than inter-subjective investigations, continuous aging rather than a particular old stage, successful case studies rather than action research; all of which are challenged in the Chinese field investigation.

The current study focuses on the topics structure, including the variables positions, connections and the vacancy of the topics. There are many Psychological variables both in English and Chinese literature, such as the degree of life satisfaction, life quality, mental health, lifespan development, subjective well-being, need, positive aging, loneliness, happiness, depression, cognition, aging Psychology, aging depression, Alzheimer disease and so on. These are all connected with the old, and some of them connected with each other. Culture, spirituality, family and other English daily concepts during 1990 -2018 web of Science occurs multiple times in the literature of psychology, but limited attention has been paid on them in Chinese literature; which might have very important implications.

After all, the Psychological research in English literature is more abundant, while the history is much longer. Because psychological terms mostly come from English, the research topics from the study of Chinese literature seems more academic, lacking key concepts from Chinese daily words, such as studies on qinqing, guanxi or relationship of the elderly in China, implicating a current important approach of Chinese aging Psychology: abandoning the local terms to adapt to globalization, which should be put right under the perspective of glocalization.

\section{REFERENCES}

[1] T. S. Wang and C.Y. Hou, "A topical structure analysis on Chinese aging study and practice in the view of culture psychology," in Proc. the Second Summit Forum of China's Cultural Psychology, 2016, pp. 164-170.

[2] A. J. Elliot, C. Sedikides, K. Murayama, A. Tanaka, T. M. Thrash, and R. R. Mapes, "Cross-cultural generality and specificity in self-regulation: Avoidance personal goals and multiple aspects of well-being in the United States and Japan," Emotion, vol. 12, pp. 1031-1040, May 2012.

[3] J. E. Gebauer, J. Wagner, C. Sedikides, and W. Neberich, "Agency-communion and self-esteem relations are moderated by culture, religiosity, age, and sex: evidence for the 'self-centrality breeds self-enhancement' principle," Journal of Personality, vol. 81, pp. 261-275, March 2013.

[4] J. Nikitin, S. Schoch, and A. M. Freund, "The role of age and motivation for the experience of social acceptance and rejection," Developmental Psychology, vol. 50, pp. 1943-1950, July 2014.

[5] F. R. Lang and L. L. Carstensen, "Time counts: future time perspective, goals, and social relationships," Psychology and Aging, vol. 17, pp. 125-139, January 2002.
[6] Library Wuhan University. Web of Science (including SCIE, SSCI, A\&HCIM CPCI). WHU in China. [Online]. Available: http://iras.lib.whu.edu.cn/ index.html

[7] L. L. Carstensen, D. M. Isaacowitz, and S. T. Charles, "Taking time seriously - A theory of socioemotional selectivity," American Psychologist, vol. 54, pp. 165-181, March 1999.

[8] R. Cabeza, "Hemispheric asymmetry reduction in older adults: The Harold model," Psychology And Aging, vol. 17, pp. 85-100, January 2002.

[9] L. L. Carstensen, "Social and emotional patterns in adulthood: Support for socioemotional selectivity theory," Psychology and Aging, vol. 7, pp. 331-338, March 1992.

[10] G. H. Elder, "Time, human agency, and social-change - Perspectives on the life-course," Social Psychology Quarterly, vol. 57, pp. 4-15, January 1994.

[11] G. W. Small, P. V. Rabins, P. P. Barry, N. S. Buckholtz, S. T. DeKosky, S. H. Ferris, and L. E. Tune, "Diagnosis and treatment of Alzheimer disease and related disorders - Consensus statement of the American Association for geriatric psychiatry, the Alzheimer's Association, and the American geriatrics society," Jama-Journal of the American Medical Association, vol. 278, no. 16, pp. 1363-1371, 1997.

[12] J. M. Perrin, M. T. Stein, R. W. Amler et al., "Clinical practice guideline: Treatment of the school-aged child with attention-deficit/hyperactivity disorder," Pediatrics, vol. 108, pp. 1033-1044, April 2001.

[13] N. L. Sin and S. Lyubomirsky, "Enhancing well-being and alleviating depressive symptoms with positive psychology interventions: A practice-friendly meta-analysis," Journal of Clinical Psychology, vol. 65, pp. 467-487, May 2009.

[14] U. Lindenberger and P. B. Baltes, "Sensory functioning and intelligence in old-age - A strong connection," Psychology and Aging, vol. 9, pp. 339-355, March 1994.

[15] P. B. Baltes, U. M. Staudinger, and U. Lindenberger, "Lifespan psychology: Theory and application to intellectual functioning," Annual Review of Psychology, vol. 50, pp. 471-507, 1999.

[16] M. M. Baltes and L. L. Carstensen, "The process of successful ageing," Ageing and Society, vol. 16, pp. 397-422, 1996.

[17] X. N. Su, The Theory and Technology on Informational Index, Beijing, PRC: Science and Technology Document Press, 2004, pp. 70-73.

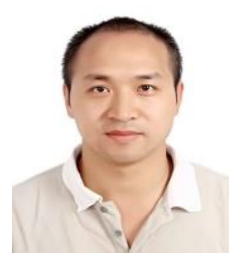

Tangsheng Wang was born in Hubei, China, on December 29th, 1981. He obtained the bachelor of arts from College of Chinese Language and Literature in Wuhan University in 2004 and the master's degree in education from Philosophy School in Wuhan University in 2006.

$\mathrm{He}$ worked in Zhejiang Ocean University as an assistant from July, 2006 to October, 2017, then worked in Jiangxi University of Technology as a lecturer till June, 2014. From then on, he studies as a PHD student of psychology in Wuhan University. He published some books or articles such as: A topical structure analysis on Chinese aging study and practice in the view of culture psychology in Tian Xie, Lisa Hale, and Jin Zhang (eds.) Proceedings of the Second Summit Forum of China's Cultural Psychology, USA: The American Scholars Press, 2016; The influence of neuroscience on modern society, Beijing in China: Peking University Press, 2012. Now his research interest is aging Psychology.

PHD T. Wang is a member of Chinese Psychological Society, and a member of Chinese Social Psychological Association.

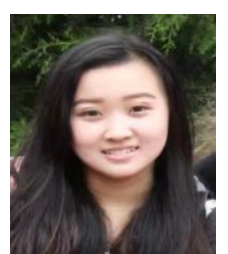

Zhiyun Yu was born in Shanghai, China, on August 30th, 2001. Currently she is studying in Harrisburg Christian School as a student. Her research interest is psycholgy. 